Case Report

\title{
Replantation of an amputated thumb: a rare case report
}

\begin{abstract}
An amputation of the thumb is a distressful injury with negative effects on one's ability to perform daily tasks, work, and support a family. It can thus impose huge psychological impacts. We report a replantation surgery in a young male patient with an amputation of the thumb at the carpometacarpal joint level. It is essential to inform healthcare professionals about the possibility of salvage. Apparently, the immediate attention of a multispeciality medical team would be necessary to increase the chance of success. This paper also reviews previous studies on the operative techniques of replantation along with their contraindications and long-term outcomes.
\end{abstract}

Keywords: Replantation; Microvascular; Thumb amputation
Volume 3 Issue 5 - 2015

\author{
Mehdi Bahari Mehrabani \\ Univercity of Medical Siences, Iran
}

Correspondence: Mehdi Bahari Mehrabani, Orthopaedic surgeon, Univercity of Medical Siences, Shahid Rajai General Hospital, Daran, Faridan, Isfahan, Iran Email Bharimehdi@yhoo.com

Received: September 06, 2014 | Published: December 09, 2015

\section{Introduction}

Replantation seeks to recover the form and function of an amputated limb by reattaching the existing neurovascular and musculoskeletal structures. Komatsu and Tamai were the first to successfully replant a human thumb in 1968. Ever since, countless detached fingers, toes, and other body parts have been effectively salvaged. ${ }^{1-3}$

However, rather than being merely determined by the recirculation to the distal part, replantation success depends on effective performance of a set of technical procedures. First, neurovascular structures need to be examined and dissected. After bone fixation, tendons and muscles have to be repaired. Microsurgical nerve repair and revascularization should then be performed and the skin needs to be properly closed. Finally, the injured hand is splinted and the circulatory changes in the thumb are carefully monitored over the postoperative period. ${ }^{4-6}$

Various parameters including the significance of the injured part, severity and mechanism of injury, and the possibility of function restoration can affect the decision to replant a severed body part. ${ }^{7}$ Efforts to salvage a body part are absolutely contraindicated if the accompanying injuries or preexisting diseases inhibit the transfer or increase the duration of the surgery. Relative contraindications to replantation are neurovascular damage at several levels mental instability. Moreover, injuries caused by guillotine are believed to have a higher chance of successful replantation compared to crush avulsion injuries. ${ }^{8,9}$

The level of injury and age are major determinants of function restoration after replantation. Replants of the fingers distal to the flexor digitorum superficialis insertion, the hand at the wrist, and the upper extremity at the distal forearm can achieve good function. ${ }^{2-4}$ Furthermore, better outcomes are expected in younger patients.

This case report describes the successful salvage of a thumb amputated at the carpometacarpal (CMC) joint level following an emergency surgery in a young male patient.

\section{Case Report}

The thumb of the non-dominant hand of an 18-year-old righthanded male patient was amputated following the explosion of fireworks. The hand was bandaged and splinted and the patient presented to the Emergency Department of Shahid Rajai Hospital within an hour of the injury. He was conscious and oriented and had a blood pressure of 110/90 mm Hg. A crush injury was evident from his stump (Figure 1). The amputated thumb was brought in a non-sterile bandage without ice.

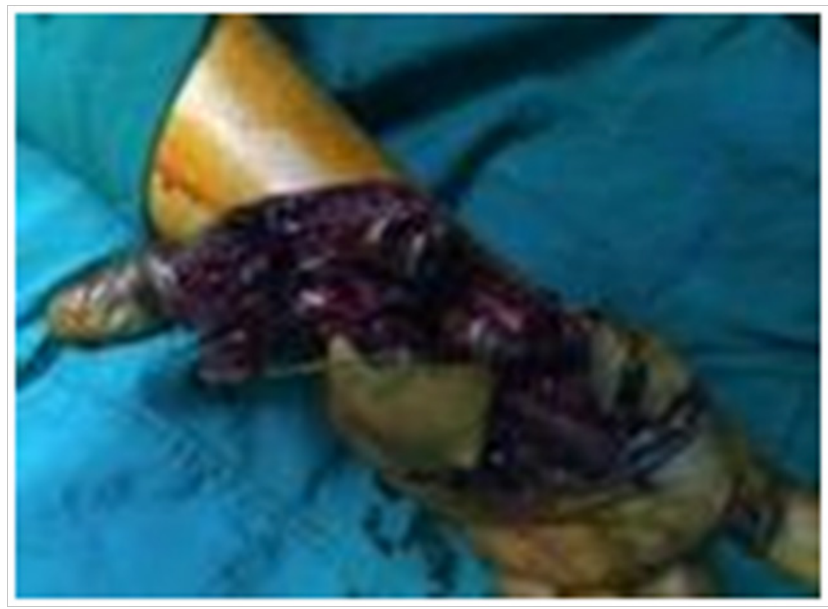

Figure I Hand stump.

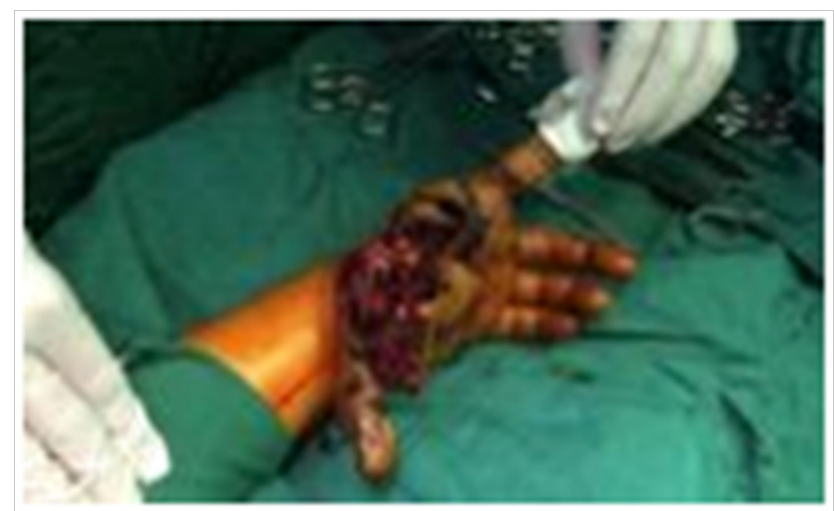

Figure 2 During surgery.

The first step was to carefully wash the amputated hand with four liters of normal saline. After thorough examinations, the therapeutic team (including an orthopedic surgeon) decided to replant the hand. The patient was hence explained about the advantages and disadvantages of the surgery and asked to provide consent. Immediately afterward, he 
was then taken up for surgery under general anesthesia. The exposed area of the amputated hand was covered with a saline-soaked gauze dressing, transferred to a sterile polythene container, and maintained kept in an ice box until delivery to the operating room (Figure 2).

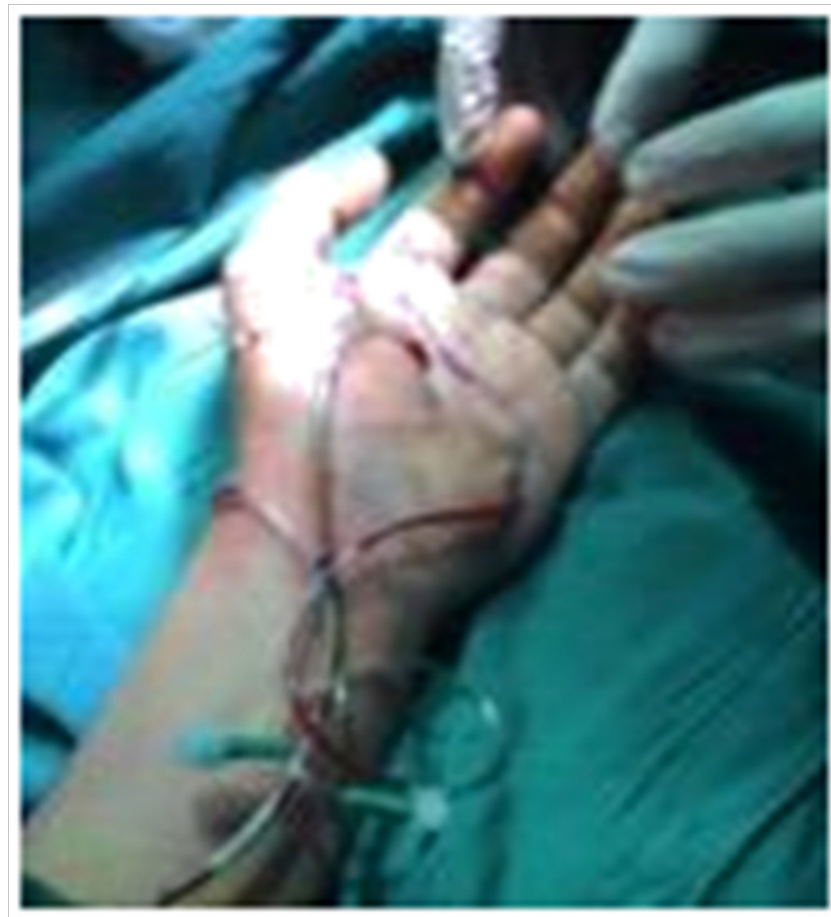

Figure 3 \& 4 After surgery slight movements were seen in his fingers.

First, the CMC joint was fixed in abduction with three $1.5 \mathrm{~mm}$ Steinmann pins. The tourniquet was elevated and the superficial and deep palmar arches and both palmar digital arteries of the thumb, along with one vein on the volar aspect, were repaired. The tourniquet was then deflated and good backflow from the two distal branches of the radial artery was observed. The tourniquet was elevated again to facilitate the repair of the superficial palmar arch at the ulnar side of the hand. Arterial input was established about four hours after the injury. The dorsal branch of ulnar nerve, the dorsoradial nerve, and the radial branch of the second digital nerve were also repaired. However, due to the extensive damage to the branch of the median nerve, it could not be initially repaired and was thus tagged to the flexor hallucis longus (FHL) tendon for future repair.

All vascular an astomoses were done with $10-0$ or 8-0 nylon sutures under naked eyes. A distal incision was made through the thenar crease to facilitate not only the identification of the distal structures, but also carpal tunnel release. In order to ensure the safe time limits, the tourniquet was frequently deflated. The tendons were repaired individually (flexor pollicis longus, extensor pollicis longus, extensor pollicis brevis, flexor pollicis brevis only deep head, AdPB only oblique head, AbPB, flexor digitorum profundus, and flexor digitorum superficialis of the second finger) or together to prevent unnecessary delays. The surgery lasted five hours and the patient recovered from anesthesia without any particular complications.

During the surgery, the patient received a single dose of low -molecular weight heparin. The same medicine was administered daily for one week. Moreover, aspirin (as an antiplatelet) and antibiotics were used for two weeks after the surgery.

The patient's vital signs and hand vascularity were carefully monitored and he was discharged after one week. Upon discharge, all his wounds were healed (Figure $3 \& 4$ ) slight movements were seen in his fingers. The patient was explained about the necessity of regular follow-up to evaluate bone and nerve healing and range of motion. He was also provided with details on the required procedures (tendon repair, tenolysis, tendon transfer, neurolysis, selective arthrodesis, and physiotherapy) over the one year after surgery.

\section{Review of Literature}

Based on the available literature, limb survival rates vary between $82 \%$ in North Carolina and $100 \%$ in Ogori Daiichi Hospital, Japan. ${ }^{10,11}$ Different scoring systems and indices such as readaptation, joint restriction, sensory recovery, and muscle strength are generally used to assess long-term outcomes. Lutz reported successful hand, finger, and toe replantation and revascularization in 64 patients with crush injuries. Follow-up of the patients for an average duration of 12 years revealed the necessity of secondary procedures in $45 \%$ of the subjects. ${ }^{12}$ In a large study, Russell et al. found $91.66 \%$ of the patients (22 out of 24) to be satisfied with both function and appearance of their replanted body parts. ${ }^{3}$ In a recent retrospective study with a mean follow-up duration of 11 years, very good/good, moderate, and poor outcomes were detected in $65 \%, 11 \%$, and $23 \%$ of the patients. More unfavorable outcomes were seen with amputations at or above the elbow level and those caused by avulsions. ${ }^{13}$

\section{Discussion}

Replantation aims to restore the function and form of a limb following a traumatic amputation. Reports from large series of patients have led to a set of recommendations for more efficient replantation. First, during patient transfer, plastic ice bags should be placed around the distal part to minimize the warm ischemia time. It is suggested to cut distal part off if it is attached to a bit of tissue. The severed part has to be covered with saline-soaked gauze, placed in a plastic bag, and maintained in ice. Instead of clamping, bleeding from the vessels of the stump needs to be managed by compressive dressing and limb elevation.

In severe injuries to the arm (from the shoulder to the wrist), the safe upper limits for warm and cold ischemia times are six and 12 hours, respectively. Nevertheless, cases of success have been documented with even longer ischemia times. ${ }^{5}$ In order to reduce ischemia times, a shunt can be inserted between the proximal and distal vessels during preoperative preparation. Moreover, better results can be expected by vascular washout with University of Wisconsin solution at $40^{\circ} \mathrm{C} .6$

Preoperative preparation should also involve fluid replacement and warming to inhibit vasoconstriction. Neurovascular structures have to be identified through making proper incisions. These structures should be meticulously tagged and protected throughout bone manipulations. Bone shortening (on the severed part not the stump) is generally necessary to decrease tension along the repaired neurovascular structures and to facilitate the closure of the injury. The existing fractures are usually fixed with K-wires.

After tourniquet deflation, the arterial flow is evaluated and additional vessel shortening is performed if inadequate proximal vessel flow is observed. The preferences of the surgeon and the prevailing clinical conditions determine the order in which various structures are repaired. In order for arterial interposition, a vein graft harvested from the contralateral arm or leg should be reversed. A successful replantation would need the restoration of at least one artery and two veins. Following revascularization, the vascularity of the muscles and the need for further debridement has to be assessed. ${ }^{7}$ 
During the postoperative period, the patient has to be provided with sufficient fluids and a warm room to minimize the risk of hypotension and vasospasm. Although some previous studies have suggested the administration aspirin, dextran, or heparin, a randomized controlled trial is warranted to confirm such suggestions. ${ }^{8,9}$ Careful monitoring of the patient's hand is essential for early detection of signs of arterial insufficiency or venous congestion. Urgent re-exploration and revision of the affected anastomosis would be crucial if any of these conditions occur.

Systemic reperfusion problems such as myoglobinuria, tachypnoea, and signs of acute respiratory distress syndrome may develop over the postoperative period. Under such circumstances, amputation would be life-saving and should be immediately performed if the patient shows progressive symptoms of reperfusion syndrome. ${ }^{7}$ Limb survival rate, function restoration, and patient satisfaction should all be considered when evaluating the outcome of surgery.

\section{Conclusion}

While the replantation techniques have evolved during the past 40 years, it is essential to inform the healthcare personnel about the possibility of saving a body part. Prompt performance of each stage will result in higher survival rates and better long-term outcomes by minimizing the ischemia time. Although further surgeries and long periods of physiotherapy may be required, a committed patient and a devoted healthcare team will boost the chance of desirable functional outcomes. In addition, the success of a particular surgery (like the one described in the current paper) can motivate the medical teams.

\section{Ackowledgments}

None.

\section{Conflicts of interst}

None.

\section{References}

1. Komatsu S, Tamai S Successful replantation of a completely cut-off thumb: case report. Plast Reconstr Surg. 1968:42:374-377.
2. May JW, Toth BA, Gardner M Digital replantation distal to the proximal interphalangeal joint. J Hand Surg Am. 1982:7(2):161-166.

3. Russell RC, O'Brien BM, Morrison WA et al. The late functional results of upper limb revascularization and replantation. J Hand Surg Am. 1984:9(5):623-633.

4. Vanstraelen P, Papini RP, Sykes PJ et al. The functional results of hand replantation. The Chepstow experience. J Hand Surg Br. 1993:18(5):556-564.

5. Wei FC, Chang YL, Chen HC et al. Three successful digital replantations in a patient after 84,86 , and 94 hours of cold ischemia time. Plast Reconstr Surg. 1988:82(2):346-350.

6. Kour AK, Phone MH, Chia J et al. A preliminary report of tissue preservation with University of Wisconsin cold storage solution in major limb replantation. Ann Acad Med Singapore. 1995:24(4Suppl): 37-41.

7. Sabapathy SR, Venkatramani H, Bharathi RR et al. Technical considerations and functional outcome of 22 major replantations (The BSSH Douglas Lamb Lecture, 2005). J Hand Surg Eur. 2007:32(5):488-501.

8. Tamai S Twenty years' experience of limb replantation-review of 293 upper extremity replants. J Hand Surg Am. 1982:7(6):549-556.

9. Goldner RD, Urbaniak JR Replantation. In: Green DP, et al. (Eds.), Green's Operative Hand Surgery (4th edn), Churchill Livingstone, New York, USA, pp. 1999:1139-1155.

10. Kleinert HE, Jablon M, Tsai TM An overview of replantation and results of 347 replants in 245 patients. $J$ Trauma. 1980:20(5):390-398.

11. Hattori Y, Doi K, To S, Oi R Results of Major Replantation of Upper Extremity. Journal of Japanese Society for Surgery of the Hand. 2002:6:863-866.

12. Lutz BS, Klauke T, Dietrich FE Late results after microvascular reconstruction of severe crush and avulsion injuries of the upper extremity. J Reconstr Microsurg. 1997:13(6):423-429.

13. Sugun TS, Ozaksar K, Ada S et al. Long-term results of major upper extremity replantations. Acta Orthop Traumatol Turc. 2009:43(3):206-213. 